\title{
Comparison of methods to calculate the Dark Current Non Uniformity
}

\author{
M.C. Ursule, Student Member, IEEE, C. Inguimbert, T. Nuns and J. Morio
}

\begin{abstract}
Analytical and Monte Carlo DCNU prediction methods are compared. The difference is studied according to the different assumptions used for the calculations. The analysis is performed as a function of the incident proton fluence. The convergence of the DCNU toward a Gaussian distribution predicted by the central limit theorem is also investigated.
\end{abstract}

Index terms - Space environment, Displacement damages, DCNU, Image sensors, Monte Carlo.

\section{INTRODUCTION}

$\mathrm{T}$ he space environment is hostile for satellites and their payload. They can suffer from significant degradations leading potentially to critical failures. Cosmic rays, radiation belts, solar flare and solar wind are sources of particles able to initiate various types of degradation in the electronic components of satellites. Energetic particles are able to reach and degrade solid state sensors (CCD, CIS) used in various applications such as star trackers, sun sensors or earth observation systems. By depositing energy in the sensor arrays, space particles cause interactions with electrons of the medium (SEE, ionizing dose) or atomic displacements (Displacement Damage Dose) [1]. Here, the creation of atomic displacements is only considered. Those displacements lead to some specific degradation in image sensors such as the increase of the dark current. This intrinsic parasitic current creates a reading noise affecting the images. The dispersion of the dark current from pixel to pixel called the Dark Current Non Uniformity (DCNU) is known to be critical for some applications that require a low noise level such as star trackers. The prediction of this degradation is essential for space missions.

The calculation of the DCNU consists in evaluating, consecutively to an irradiation by incident particles (protons, electrons, neutrons), the dark current induced by the amount of damage produced in each pixel of an array. For each pixel the damage is the sum of the damage produced by different interactions. Each interaction initiates a damage cascade depending on the energy of the Primary Knock on Atom (PKA). The probability to get a given amount of damage is a combination of the probability to get a recoil atom of a given energy, by the amount of atomic displacements produced by this PKA. This probability is known as the individual Probabiliy Density Function (individual PDF). At the array of pixels level, the convolution of the damage produced by different PKAs can be solved by a Monte Carlo algorithm. That means, randomly selecting the energy of the PKA in the differential interaction cross section [2, 4], and calculating for each PKA the amount of produced damage. This calculation is

1) M.C. Ursule (PHD student), C. Inguimbert, T. Nuns and J. Morio are with ONERA-DESP, 2 avenue E. Belin, 31055 Toulouse, France (tel: 33 562252734, email: marie-cecile.ursule@onera.fr). repeated for each pixel, according to the number of interactions that have occurred in each pixel. But this Monte Carlo algorithm [2-4] is CPU time consuming, and this convolution can be performed [5-12] by a direct numerical integration [13]. Such alternative way of calculation has been developed first, and was very successful over the years. This method has the benefit to be very fast, if the product of convolution leads to a known function, avoiding this way to perform the convolution product itself. It is why, originally, Gaussian functions [5-7] were used to describe the differential damage distribution of the individual interactions (individual PDF). But, Gamma functions have also been adopted [8-10], because at low fluence level, they better represent the differential interaction cross sections.

Analytical and Monte Carlo calculation methods are thus equivalent in the limit of the assumptions made to simulate the differential interaction probabilities. The impact of the use of different probability density functions, on the DCNU calculation shall be investigated. This paper aims at evaluating this impact by comparing the full Monte Carlo calculation with some methods based on the use of approximated PDF. The differences will be analyzed according to the incident fluence. In addition, at high fluence level, the DCNU converge towards a Normal distribution having a known mean and standard deviation. But this speed of convergence is closely dependent on the chosen differential interaction probability. This will also be analyzed. However, it was noted that, all these methods do not take into account some physical processes such as the Electric Field Enhancement effect (EFE), or the impact of the Total Ionizing Dose (TID) on the DCNU. These degradation mechanisms, that are neglected by the different methods will, therefore, not be discussed in this work.

For this study, a method based on the same assumption as the methods of references [6-8] have been developed and compared to the full Monte Carlo calculations. The experimental data of the Jade device [14] from e2v will serve as reference for comparison.

In the first part, both the analytical and Monte Carlo DCNU models will be presented. Then, the second part will be devoted to the description of the method implemented in our toolkit. The third part shows a comparison of these different methods of calculation. The limits beyond which the DCNU converge to a normal distribution having a known mean and standard deviation will also be established.

\section{DCNU METHODS OF PREDICTION}

\section{A. Monte Carlo approach}

A priori, the most straightforward way to predict the DCNU consists in a Monte Carlo algorithm [2-4] that directly reproduces the damage process. But historically, because the Monte Carlo method is CPU time consuming, faster alternate 
ways were preferred [5-12]. The principle of these two approaches is similar but the methods of calculation differ slightly [13]. The method of prediction based on the Monte Carlo algorithm used in this study has been presented and compare to experimental data in detail in [3, 4], but for the sake of clarity and the understanding of the study, the key points are reminded briefly here (cf. Fig. 1, Monte Carlo method). According to the incident fluence a different number of interactions (Coulombic, nuclear elastic and nuclear inelastic) is produced in each pixel. The distribution of this number of interactions follows a Poisson distribution at low fluence levels and then tends to a normal distribution for higher fluences with an average $\lambda$ :

$$
\lambda=\sigma_{c s} \Phi \eta V_{\text {pixel }} R
$$

Where $\eta$ is the atomic density of the target material (atom/ $\left.\mathrm{cm}^{3}\right), V_{\text {pixel }}$ the volume of the pixel $\left(\mathrm{cm}^{3}\right)$ and $R$ the proportion of interaction produced in the pixels that lead to a degradation of the depleted region. Each interaction will produce a Primary Knock on Atom PKA of a given energy $[\mathrm{keV}, \mathrm{MeV}]$ that will initiate a damage cascade resulting in a given number of atomic displacements. The DCNU can be estimated by defining randomly for each pixel the number of interactions $\lambda_{i}$, and then evaluating the number of atomic displacements generated by each interaction with a second random draw. The number of atomic displacements generated by each interaction is then evaluated randomly. Thanks to the characteristics of the recoil nuclei of coulombic interactions (low energy $\sim \mathrm{keV}$, short range), the number of atomic displacements can be deduced with the Lindhard energy partition function after estimation of the recoil energy of the PKA [15]. For nuclear interactions, because of the recoil nuclei range, a GEANT4 application $[16,17]$ has been developed to model the non-negligible number of displacement cascades entering in the depleted volume after being initiated outside. This application models and records in a database the displacement cascades that spread over neighbor pixels created by thousands of interactions [16]. In a given pixel, the number of atomic displacements created will be randomly selected in this database. This method allows taking into account border crossing effects. But, previous works show that the border crossing effect is negligible when the ranges of recoil ions are much smaller than pixel dimensions [4]. However, if the dimensions of the depleted volume are in the order of the micrometer, this effect needs to be considered in the simulations [4]. Finally, the dark current is estimated by assuming its proportionality to the number of atomic displacement thanks to the use of the Universal Damage Factor (UDF) $K_{\text {dark }}$ [18]. Mechanisms such as Electric Field Enhancement effect (EFE), or the Total Ionizing Dose (TID) is not taken into account in this study.

\section{B. "Analytical" method}

As described in the previous section, many random draws, in both the interaction cross sections and the Poisson's law are required for the full Monte Carlo algorithm calculation. But this convolution product can be performed thanks to a direct numerical integration [13]. The DCNU is the result of the convolution by itself of the probability density function of the number of displacements generated by a single interaction (individual PDF). The individual PDF is a function of both the interaction cross section and the Lindhard energy partition function. The distribution of damage of a population of pixels undergoing $\lambda_{i}$ interactions is the $\lambda_{i}$ convolution of the individual PDF. As the distribution of the population of pixels undergoing $\lambda_{i}$ interactions is governed by a Poisson's law, the final DCNU is simply the sum of the distributions previously calculated for different $\lambda_{i}$ values, weighted by this Poisson's law. Historically this way of calculation has been developed first [5-7] and have been very successful along the years [812]. A detailed description can be found in ref. [7] (Fig. 1a \& $1 \mathrm{~b}$ in [7]). The salient points of this method are reminded here. It differs from the Monte Carlo approach only by the assumption made on the shape of the individual PDF (Gamma, Gaussian) used to calculate the number of displacements generated by a single interaction. The analytical convolution product also does not take into account the existing statistical noise of this stochastic damage process. But, the convolution product leads to an exact distribution neglecting any statistical noise, toward which the DCNU tends at very high fluence level. In return, this method is significantly faster than the Monte Carlo one, explaining its success over the years.

If the number of interaction per pixel $\lambda_{i}$ is big enough, the damage produced becomes proportional to the Non Ionizing Energy Loss (NIEL) of the incident particle. The average number of interactions in a pixel is given by the following formula:

$$
N=\sigma_{c s} \rho \Phi V_{D} \frac{N_{A}}{A}
$$

Where $\sigma_{c s}$ is the interaction cross section $\left(\mathrm{cm}^{2}\right), \rho$ the density of silicon $\left(\mathrm{g} / \mathrm{cm}^{3}\right), \Phi$ the incident fluence (particle $/ \mathrm{cm}^{2}$ ), $V_{D}$ the depletion volume $\left(\mathrm{cm}^{3}\right), N_{A}$ Avogadro's number $\left(\mathrm{mol}^{-1}\right)$ and $A$ the atomic mass of the target material $(A=$ $28 \mathrm{~g} / \mathrm{mol}$ for silicon).

The mean and the standard deviation of the individual PDF will be called respectively $\mu_{\text {disp }}$ and $\sigma_{\text {disp }}$ in the rest of this paper. Each interaction (Coulombic, nuclear elastic and inelastic) are treated separately with different individual PDF. In the case of elastic Rutherford scattering, considering a high number of coulombic events per pixel, Marshall et al. approximated the individual PDF by a Gaussian distribution of mean $\mu_{\text {disp }}$ and variance $\sigma_{\text {disp. }}^{2}$. As we will see further in the paper, this approximation is only valid when the average number of interactions per pixel is large enough so that the final DCNU tends also to a Normal law (appendix A \& B). In that case where a large number of interactions is produced, Marshall $[6,7]$ shows that the convoluted damage distribution can tend to a Gaussian distribution defined by a mean which is the product of the average number of interactions and the mean of this individual PDF $\left(N \mu_{\text {disp }}\right)$, and a variance which is the product of the average number of interactions and the variance of this individual PDF $\left(N \sigma_{\text {disp }}^{2}\right)$ (Demonstration in appendix A \& B) $[6,7]$ (appendix A \& B). But, before converging towards a Normal law the DCNU is driven by the shape of the Rutherford scattering density probability function. Robbins [8] proposed to simulate the individual PDF with a gamma 
The first and second moment of the individual PDF $\left(\mu_{\text {disp }}\right.$, $\left.\sigma_{\text {disp }}\right)$ are proportional respectively to the mean deposited energy $E_{d e p}$ to create atomic displacements and its standard deviation. For coulombic interactions, it has been evaluated thanks to the NIEL of NEMO code [19]. The number of atomic displacements is simply deduced knowing the threshold displacement energy $T_{d}$ according to the formula 1 . For nuclear interactions, these momentums that correspond to the nuclear NIEL are estimated thanks to a database evaluated with GEANT4 (DBG4, cf. equation 2).

$$
\begin{aligned}
& \mu_{\text {disp }}=\frac{\mathrm{E}_{\mathrm{dep}}}{\eta \sigma_{c S} W} \frac{\rho W}{2 T_{d}} \quad \text { and } \quad \sigma_{\text {disp }}=\frac{\sigma_{E_{\text {dep }}}}{\eta \sigma_{c S} W} \frac{\rho W}{2 T_{d}} \\
& \mu_{\text {disp }}=<D B G 4>\text { and } \sigma_{\text {disp }}=\sigma_{D B G 4}
\end{aligned}
$$

Table 1: Characteristics of the simulations realized in this study. For an (2) elastic) and the mean number of coulombic interactions per pixel are

threshold energy $(\mathrm{MeV})$.

\section{B. method 2, limit at high fluence level}

One can show (appendix VI.B) that the result of the method 1 is a DCNU of a known mean $\lambda_{\mu \text { disp }}$, and standard deviation $\sqrt{\lambda \sigma_{\text {disp }}^{2}+\lambda \mu_{\text {disp }}^{2}}$. But the shape of this distribution is not a priori known. It is affected by the shape of both the chosen individual PDF and the Poison's law. But, when the incident fluence increases, or in a large geometry, the number of interaction per pixel $\lambda_{i}$ reaches a level for which some statistical simplifications can be applied. In that case, for each pixel, according to the central limit theorem and regardless of the shape of the individual PDF, the damage distribution on the pixel array tends to be normally distributed around this average value $\lambda \mu_{\text {disp }}$, and having a standard deviation $\sqrt{\lambda \sigma_{\text {disp }}^{2}+\lambda \mu_{\text {disp }}^{2}}$ ([8] and proof in the appendix VI.A). This distribution is noted D3 in Fig. 1 (method 2). The method 2 is simply a random draw within the $D 3$ distribution, to take into account the statistical noise which otherwise does not appear in this theoretical distribution.

For lower fluences, below the convergence level of the DCNU towards a Gaussian distribution, the discrepancy between the DCNU and a Gaussian distribution has been investigated. Thus, for the same reasons than in the alternative method 1 , both Gamma and Gaussian distributions have been studied. In the next section, both methods $1 \& 2$ will now be compared to the Monte Carlo approach. Some measurements performed on the Jade device will also serve as reference.

\section{COMPARISONS OF THE PREDICTION METHODS}

A comparison was made between the Monte Carlo method, and the two simplified approaches 1 and 2. Comparisons with experimental measurements performed on JADE (E2V) devices [20] are also made. The fluence has been adapted to vary the average number of interaction per pixel $\lambda$ in the range $[0.381,5531]$. Four energy and five fluences per energy were then considered (cf. Table 1). energy, the fluence, the mean number of nuclear interactions (inelastic and presented. For coulombic interactions the cross sections have been calculated according to the ZBL model [21]. Both nuclear elastic and inelastic cross sections are extracted from GEANT4 [16, 17].

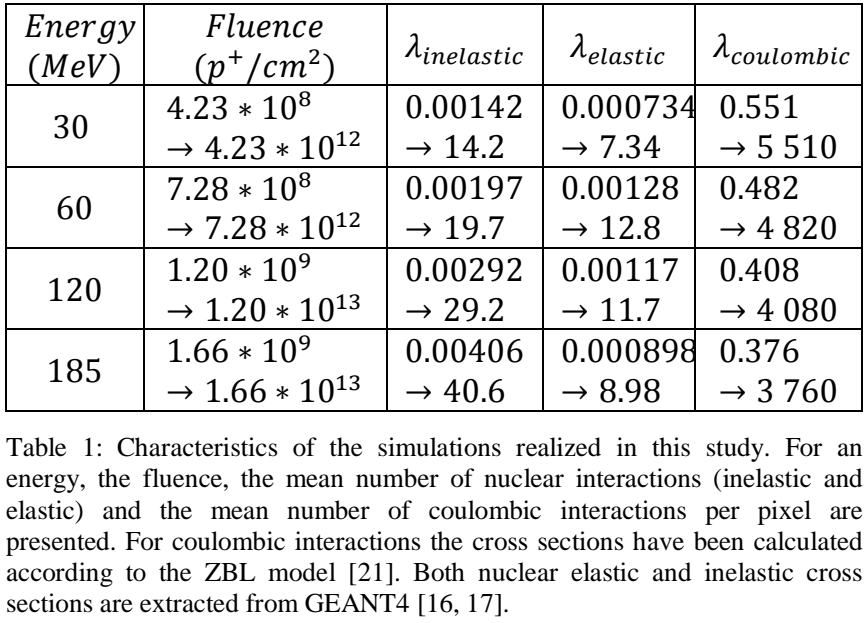

As explained in the previous sections, the shape of the final DCNU is a compromise between the individual PDF chosen for the calculation and the speed of convergence of the DCNU towards a Normal distribution. Two types of functions (Gaussian, Gamma) have been tested for individual PDF to confirm the interest of gamma distribution raised by Robbins [8]. The contributions of the three interactions have been considered but the paper presents only the results of Coulombic and inelastic interactions. Indeed, the case of nuclear elastic is quite identical to the degradation caused by nuclear inelastic interactions. Moreover, only DCNU generated by $60 \mathrm{MeV}$ protons are presented because the trend is the same for other irradiation energies. All these prediction methods have a completely different CPU time. Simplified methods 1 and 2 estimate the DCNU of a sensor array of 500000 pixels in a few minutes regardless the fluence, when the Monte Carlo approach may need several hours or days for the most critical configurations (i.e. large fluences or large geometries). The Coulombic interactions are the most CPU time consuming, as several thousand of interactions can occur per pixel. The results are presented according to three groups: large fluences generating in average thousands of interactions per pixel, intermediary fluences generating in average between 1 and 1000 interactions per pixel, and weak fluences generating in average less than a single interaction per pixel.

\section{A. High fluence regime}

For the largest fluences considered in this study $(60 \mathrm{MeV}$, $\left.7.28 * 10^{12} \mathrm{p}+/ \mathrm{cm}^{2}, \lambda_{\text {inelastic }}=19.7, \lambda_{\text {coulombic }}=4820\right)$, the three methods converge toward the same Gaussian distribution (cf. Fig. 2). Those largest fluences were considered in order to work with $\lambda$ close to those used in the literature with analytical methods. The use of a normal distribution instead of a gamma distribution for both methods (1 and 2) provides, at low degradation level, worse results than the use of a gamma distribution. This phenomenon can be observed for both the Coulombic and nuclear inelastic interactions (Fig. 3 \& Fig. 4). Surprisingly, even if the average number of nuclear interactions per pixel remains relatively small $\left(\lambda_{\text {inelastic }}=19.7\right)$, the final degradation distribution tends to a Gaussian (Fig.4). The statistical noise at low damage level (Fig.4) is due to this relatively low value of $\lambda_{\text {inelastic. }}$ 


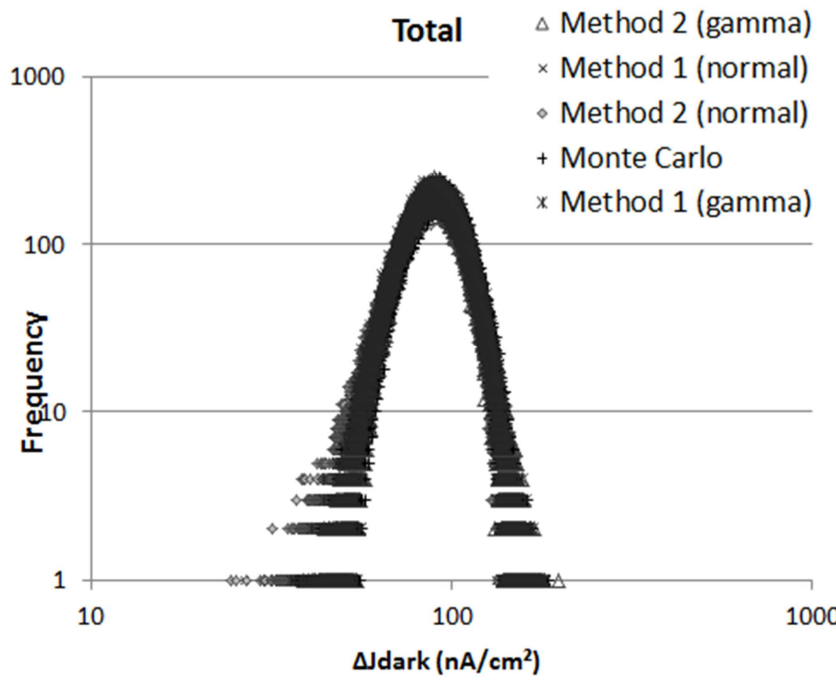

Fig. 2: Total degradation generated by an incident flux of protons $(60 \mathrm{MeV}$; $\left.7.28 * 10^{12} p+/ \mathrm{cm}^{2}\right)$.

\section{Coulombic interactions}

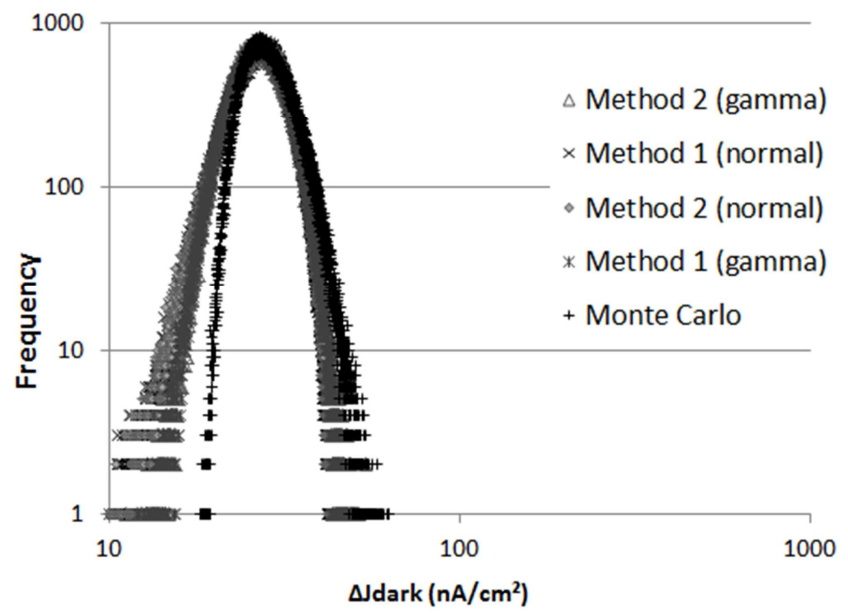

Fig. 3: Degradation caused by coulombic interactions for an incident flux of protons $\left(60 \mathrm{MeV} ; 7.28 * 10^{12} \mathrm{p}+/ \mathrm{cm}^{2}\right)$ with $\lambda_{\text {coulombic }}=4820$.

\section{Nuclear elastic interactions}

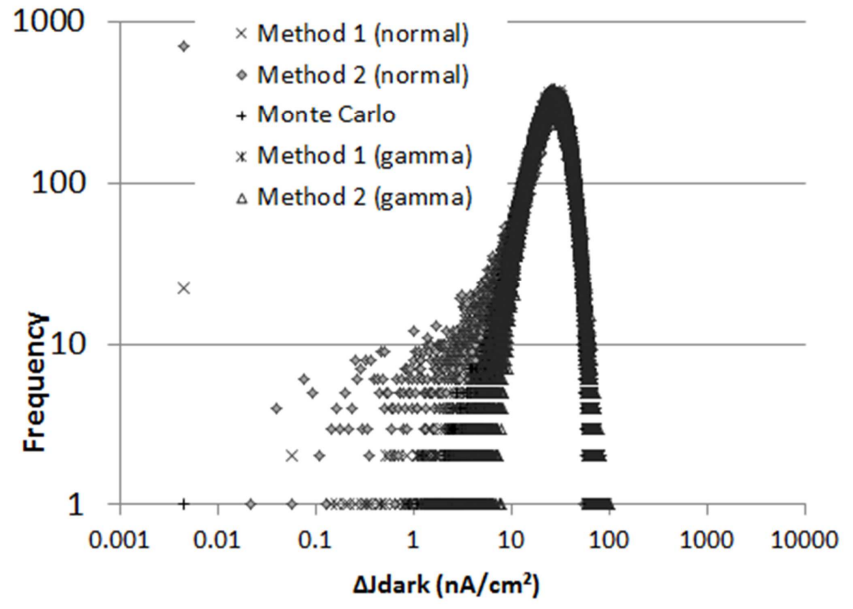

Fig. 4: Degradation caused by nuclear interactions for an incident flux of protons $\left(60 \mathrm{MeV} ; 7.28 * 10^{12} \mathrm{p}+/ \mathrm{cm}^{2}\right)$ with $\lambda_{\text {inelastic }}=19.7$.
The convergence speed towards a Gaussian distribution depends on the shape of the individual PDF. It is thus different for each type of interactions. This can be demonstrated by the inequality of Berry Esseen [21]. The Berry Esseen inequality allows the quantification of the discrepancy between the distribution of the individual PDF and a normal distribution:

$$
\begin{gathered}
\sup _{x}\left|P\left(\frac{S-\lambda \mu_{\text {disp }}}{\sqrt{\lambda\left(\sigma_{\text {disp }}^{2}+\mu_{\text {disp }}^{2}\right)}}<x\right)-\xi(x)\right| \leq \frac{C L_{1}^{3}}{\sqrt{\lambda}} \\
L_{1}^{3}=\frac{\mathbb{E}\left|X_{1}\right|^{3}}{\left(\mu_{\text {disp }}^{2}+\sigma_{\text {disp }}^{2}\right)^{3 / 2}}
\end{gathered}
$$

Where $\xi(x)$ is the cumulative distribution function of a normal random variable, $C$ a constant (here equal to 0.7655 according to [22]) and $E\left|X_{1}\right|^{3}$ the third order moment. The right-hand term is an upper bound of the discrepancy between the modelled distribution and the Gaussian distribution. The different convergence speeds have been evaluated by calculating $\frac{C L_{1}^{3}}{\sqrt{\lambda}}$ for different $\lambda$. The more this term tends to 0 , the more the distribution tends to a Gaussian distribution $(0=$ Gaussian). The calculation of this limit (cf. Table 2 ) proves that the nuclear inelastic and elastic interactions converge faster than coulombic interactions towards a normal distribution. In order to obtain a coulombic limit equivalent to a nuclear limit, $\lambda_{\text {coulombic }}$ needs to be a thousand time higher than $\lambda_{\text {nuclear }}$. The speed of convergence is closely dependent on the asymmetry of the individual PDF. The differential Rutherford interaction cross section is a very steep decreasing function while the energy distribution of inelastic recoil nuclei looks more like a Gaussian. That explains the good speed of convergence of the inelastic interactions. For this level of fluence, the simplified methods 1 and 2 give an accurate prediction of the DCNU faster than the Monte Carlo approach.

\begin{tabular}{|c|c|c|c|}
\hline$\lambda$ & Inelastic & Elastic & Coulombic \\
\hline 0.001 & 44.4 & 38.1 & 1484 \\
\hline 0.01 & 14.0 & 12.0 & 469 \\
\hline 0.1 & 4.44 & 3.81 & 148 \\
\hline 1 & 1.40 & 1.20 & 46.9 \\
\hline 10 & 0.444 & 0.381 & 14.8 \\
\hline 100 & 0.140 & 0.120 & 4.69 \\
\hline 1000 & 0.0444 & 0.0381 & 1.48 \\
\hline 10000 & 0.0140 & 0.0120 & 0.469 \\
\hline
\end{tabular}

Table 2: Right-hand term of the Berry Esseen inequality for the three interactions in function of the mean number of interactions per pixel, $\lambda$.

\section{B. Intermediary fluence regime}

When the fluence and accordingly the number of interactions per pixel decrease $\left(\lambda_{\text {inelastic }}=0.197\right.$ and $\lambda_{\text {coulombic }}=48.2$ at $\left.60 \mathrm{MeV}, 7.28 * 10^{10} \mathrm{p}+/ \mathrm{cm}^{2}\right)$, the use of these two different PDF (Gaussian , Gamma) lead to different results. But, methods 1 and 2 give results relatively close to each other (Fig. $5)$. 


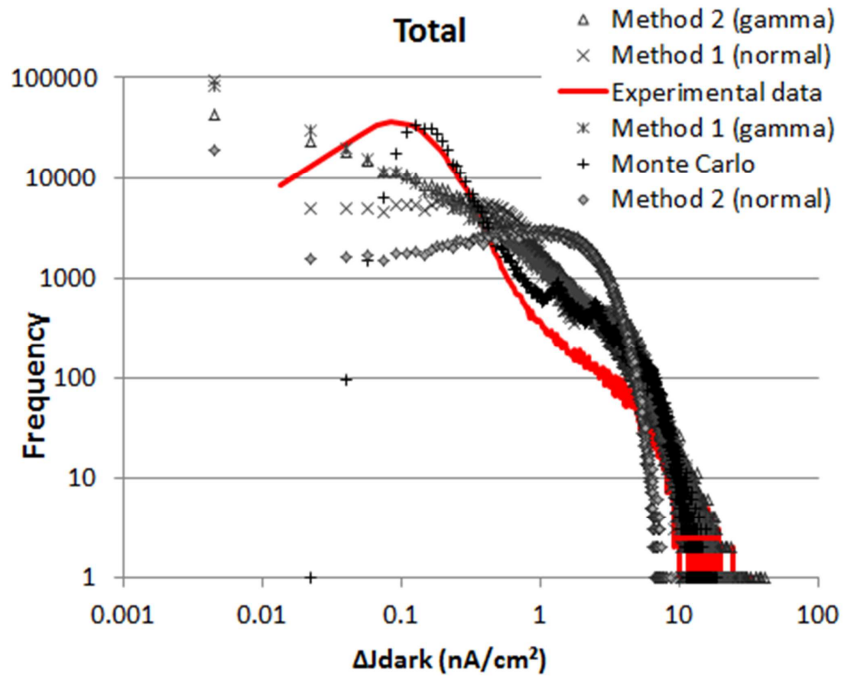

Fig. 5: Total degradation generated by an incident flux of protons $(60 \mathrm{MeV}$; $\left.7.28 * 10^{10} \mathrm{p}+/ \mathrm{cm}^{2}\right)$. Experimental data are from ref. [20].

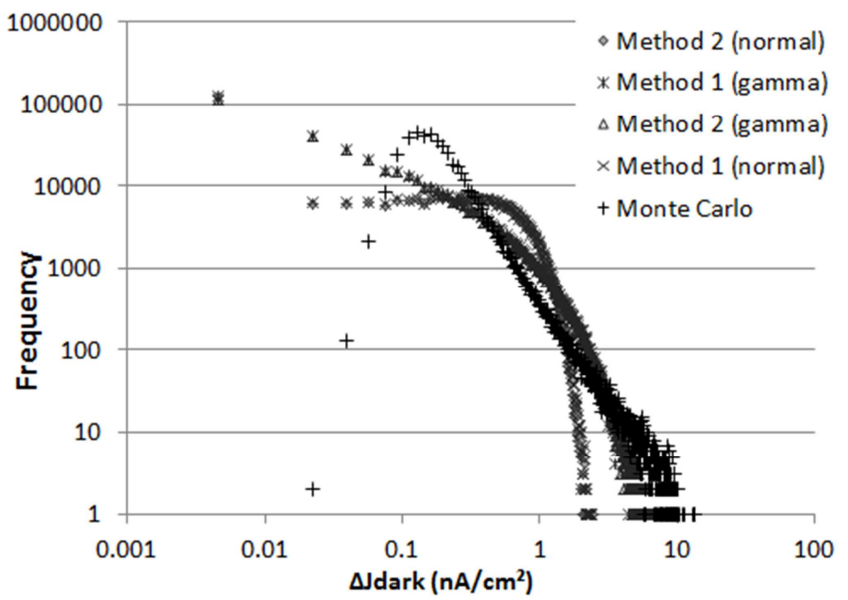

Fig. 6: Degradation caused by coulombic interactions for an incident flux of protons $\left(60 \mathrm{MeV} ; 7.28 * 10^{10} \mathrm{p}+/ \mathrm{cm}^{2}\right)$ with $\lambda_{\text {coulombic }}=48.2$.

\section{Nuclear elastic interactions}

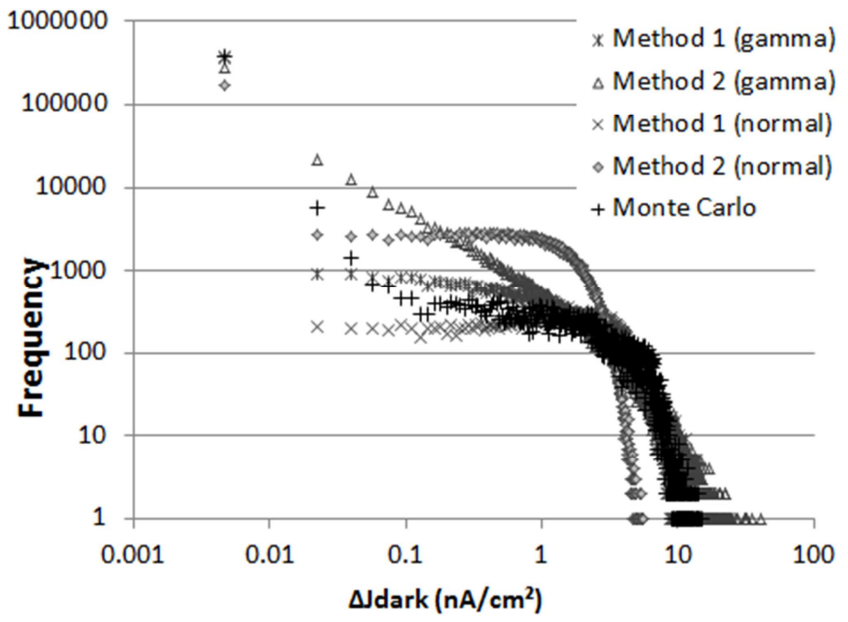

Fig. 7: Degradation caused by nuclear inelastic interactions for an incident flux of protons $\left(60 \mathrm{MeV} ; 7.28 * 10^{10} \mathrm{p}+/ \mathrm{cm}^{2}\right)$ with $\lambda_{\text {inelastic }}=0.197$.
These two methods experience difficulty to predict both Coulombic and nuclear contributions (Fig. 6 \& Fig. 7). In particular this is true for the low degradation regime $\left(\Delta J_{\text {dark }}<\right.$ $1 \mathrm{nA} / \mathrm{cm}^{2}$ ). The methods based on normal distributions underestimate the number of hot pixels in regards to the other methods. The sampling of simplified methods seems to be too small to predict the degradation at this level of fluence. The Kullback-Leibler (KL) divergence method [23] has been used to compare these three estimations with experimental data (Fig. 5). The criterion of comparison chosen for the comparison and which highlight the dissimilarity between the different distributions is the distance between the reference case $P(x)$ (here the experimental results) and the modeled distribution $Q(x)$ :

$$
D_{K L}(P \| Q)=\sum_{x} P(x) \log \frac{P(x)}{Q(x)}
$$

Two identical distributions will lead to a comparison criterion $D_{K L}(P \| Q)=0$. The smaller the $D_{K L}(P \| Q)$ parameter is, the closer the simulated $Q(x)$ distribution is close to the experimental $P(x)$ distribution. The Fig. 8 compares the divergence for each prediction method proposed in our study. The four first points of the simulated distributions have not been considered for the calculation as they are very badly estimated (Fig. 5). At this damage level, the ionizing dose is significant and affects the DCNU. The analysis is focused on the tail of the DCNU. As can be seen in Fig. 8, the Monte Carlo approach with an average value of $D_{K L}(P \| Q)<0.16$ provides the results in best agreement with experimental data. This is true whatever the incident energy for a fluence level of $7.28 e 10 p+/ \mathrm{cm}^{2}$ that corresponds to $\lambda \sim 50$. At this level of fluence, the Monte Carlo approach gives the most relevant prediction.

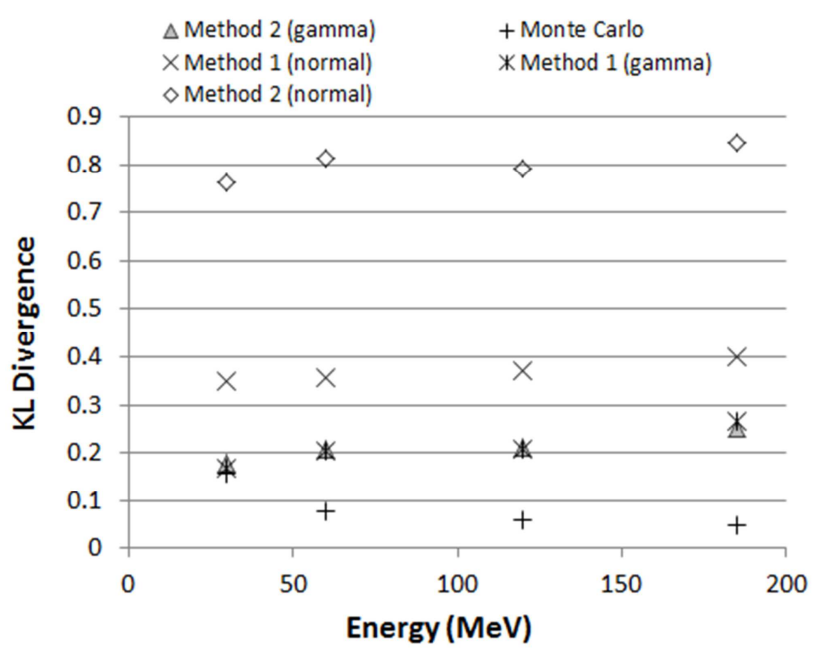

Fig. 8: Calculation of the Kullback-Leibler divergence of the predicted results with experimental data at four proton energies $(30 \mathrm{MeV}, 60 \mathrm{MeV}, 120 \mathrm{MeV}$, $185 \mathrm{MeV}$ ) and for an incident flux of $7.28 * 10^{10} \mathrm{p}+/ \mathrm{cm}^{2}$.

\section{Low fluence regime}

For weak fluence (i.e. $\lambda<1$, cf. Fig. 9) and despite an average number of interactions per pixel relatively small $\left(\lambda_{\text {coulombic }}=\right.$ 0.482 at $60 \mathrm{MeV}, 7.28 * 10^{8} \mathrm{p}+/ \mathrm{cm}^{2}$ ), the Monte Carlo approach and the simplified methods based on gamma distribution give surprisingly quite similar DCNU predictions. 
Total

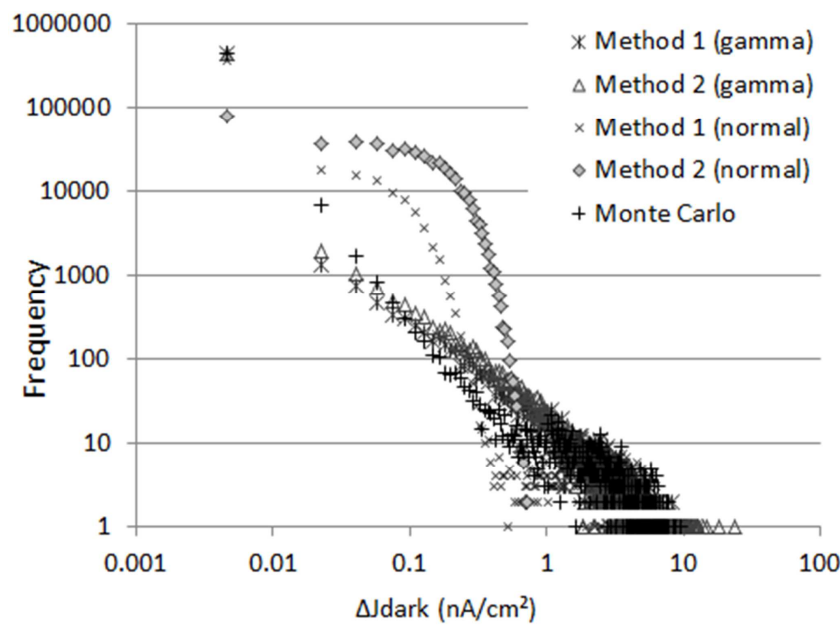

Fig. 9: Total degradation generated by an incident flux of protons $(60 \mathrm{MeV}$; $\left.7.28 * 10^{8} \mathrm{p}+/ \mathrm{cm}^{2}\right)$.

\section{Coulombic interactions}

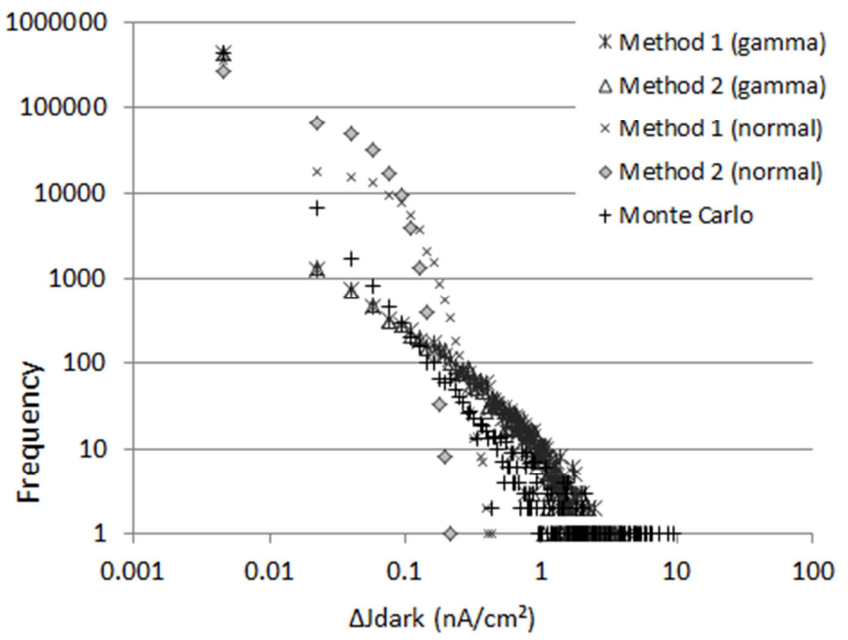

Fig. 10: Degradation caused by coulombic interactions for an incident flux of protons $\left(60 \mathrm{MeV} ; 7.28 * 10^{8} \mathrm{p}+/ \mathrm{cm}^{2}\right)$ with $\lambda_{\text {coulombic }}=0.482$.

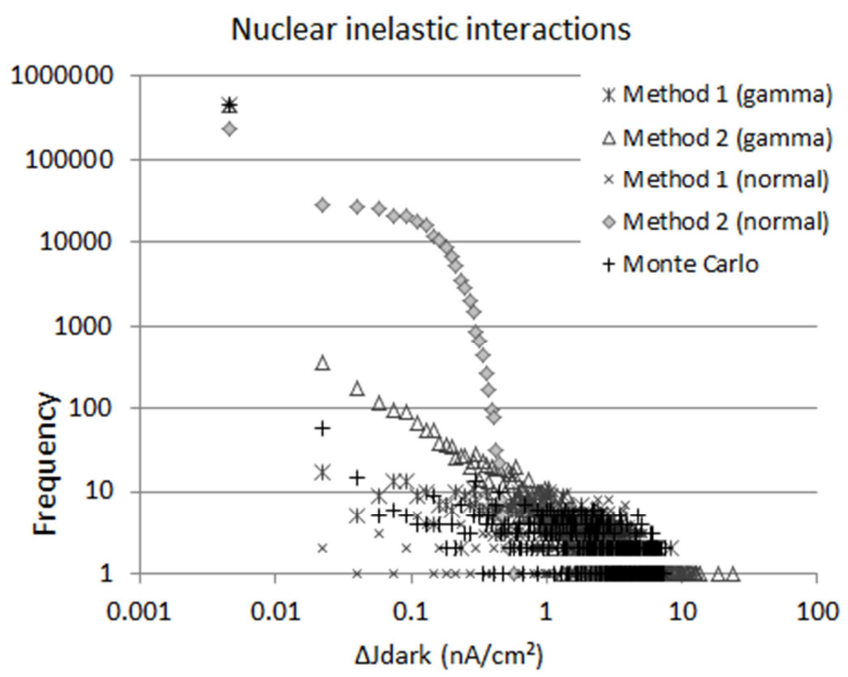

Fig. 11: Degradation caused by nuclear inelastic interactions for an inciden flux of protons $\left(60 \mathrm{MeV} ; 7.28 * 10^{8} \mathrm{p}+/ \mathrm{cm}^{2}\right)$ with $\lambda_{\text {inelastic }}=0.00197$.
At this low fluence level, when $\lambda<1$, the final DCNU tends towards the individual PDF. For example, when $\lambda_{\text {Coulombic }}=$ 0.482 , the number of nuclear interactions becomes very low $\left(\lambda_{\text {Nuclear }}=0.00197\right)$, and the shape of the DCNU is driven by the shape of the Coulombic PDF (Fig. $9 \&$ Fig. 10). For coulombic interactions the individual PDF can be calculated according to the ZBL interaction cross section [21] and the Lindhard energy partition function [16]:

$$
\propto \frac{d \sigma_{Z B L}}{d Q} \cdot \frac{d Q}{d G(Q)}
$$

Where $\mathrm{Q}$ is the recoil energy of the PKA, $\mathrm{d} \sigma_{\mathrm{ZBL}} / \mathrm{dQ}$ the differential ZBL cross section, and $\mathrm{G}(\mathrm{Q})$ the Lindhard energy partition function. The ZBL differential distribution is a strongly decreasing function comparable to a gamma function. That explains why using a gamma function in the analytical methods (1\&2) gives results close to the full Monte Carlo algorithm (Fig. 9, Fig. 10). For nuclear interactions (Fig. 11), the agreement is better when using a Gaussian PDF that better represents the shape of the differential nuclear distribution.

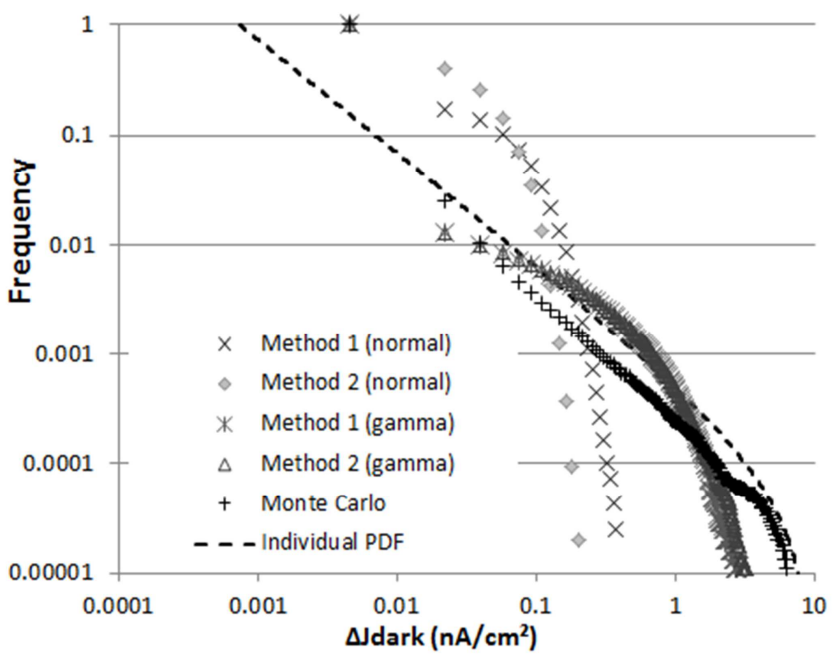

Fig. 12: Comparison between DCNU and individual PDF caused by coulombic interactions $\left(\lambda_{\text {coulombic }}=0.482\right)$. Those distributions have been converted, normalized and integrated for the comparison.

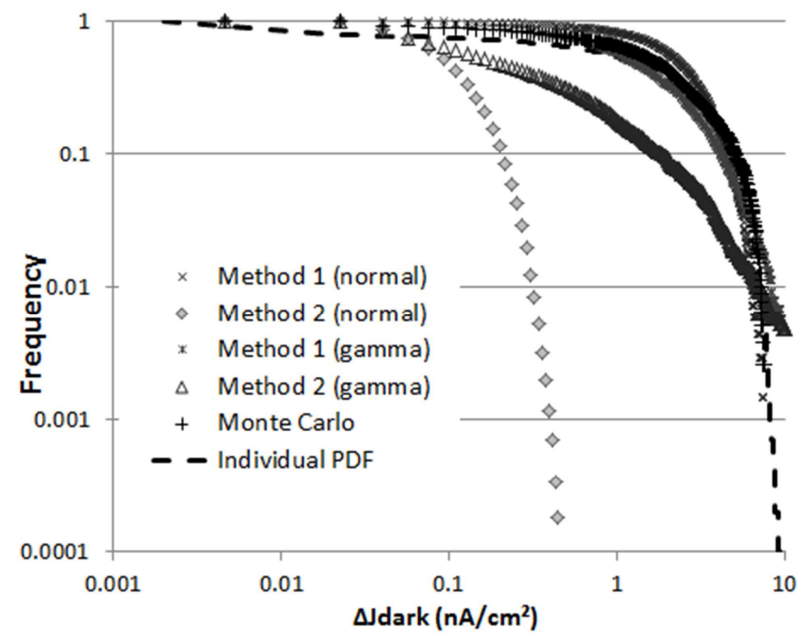

Fig. 13: Comparison between DCNU and individual PDF caused by nuclear interactions $\left(\lambda_{\text {nuclear }}=0.00197\right)$. Those distributions have been converted, normalized and integrated for the comparison. 
In order to be compared, the individual PDF and the total degradations predicted by the three approaches have been converted into integrated probabilities (cf. Fig. 12 and Fig. 13). As we can see on the Fig. 12 and Fig. 13, the final DCNU and individual PDF are superimposed on most of the dark current range. It shows the very good agreement between the full Monte Carlo algorithm and the simplified approaches based on the use of a gamma function. On the contrary, the use of a Gaussian distribution is not relevant (Fig. 12). In conclusion, the good agreement between the method 1 and the Monte Carlo approach shows that a Gamma function is well suited to represent the individual PDF for Coulombic scattering (Fig. 10).

\section{CONCLUSION}

Analytical DCNU calculation methods, that have been widely used in the past has the benefit to be faster than Monte Carlo algorithm. But they make some assumptions on the used damage probability density function that impacts the final result. In other words, such kind of methods are equivalent to the Monte Carlo approach in the limit of the made assumption. In this work, the impact of the use of different probability density functions on the DCNU calculation has been studied. The analysis has been performed in term of different incident fluence levels.

It is demonstrated that the DCNU is a distribution having a known mean and standard deviation (resp. $\lambda_{\mu \text { disp }}$, $\sqrt{\left.\lambda \sigma_{\text {disp }}^{2}+\lambda \mu_{\text {disp }}^{2}\right)}$. This distribution tends towards a Gaussian function at high fluence level $\left(\lambda_{\text {inelastic }} \sim 10\right.$, $\left.\lambda_{\text {coulombic }} \sim 1000\right)$. At low fluence regime $\left(\lambda_{\text {coulombic }}<1\right.$ the shape of the DCNU is driven by the Coulombic probability density function. In between the DCNU can be well simulated using a Gamma function as a probability density function. This explains in particular the decreasing exponential often observed experimentally on the tail of the DCNU.

A difference of convergence speed towards a Gaussian distribution is observed as a function of the interaction type. The nuclear interactions need a $\lambda$ value a thousand times smaller than the coulombic interactions to converge towards a Gaussian distribution. But when the fluence decreases (i.e. $1<\lambda<1000$ ), some discrepancies between methods appear. At this fluence level, the Monte Carlo approach provides the best results according to the calculation of the KullbackLeibler divergence. The simplified methods based on gamma distribution underestimate the low degradation levels, and the simplified methods based on normal distribution overestimate the pixels lightly impacted and underestimate the hot pixels. At weak fluences (i.e. $\lambda<1$ ), the prediction made using a gamma PDF are relatively close to the Monte Carlo simulations showing that Gamma functions are well suited to represent Coulombic differential interaction probabilities.

\section{APPENDIX}

\section{A. Demonstration of the method 2}

This demonstration determines the shape, the mean value and the variance of the distribution $D 3$ used in the alternative method 2. The goal is to estimate the distribution of the random variable $\mathrm{S}$ defined as $\mathrm{S}=\sum_{j=1}^{\lambda_{i}} X_{j}$ where the random variable $\lambda_{i}$ follows a normal distribution with mean $\lambda$ and $X_{j}$ are independent and identically distributed (i.i.d.) random variables as $X$ with mean $\mu_{\text {disp }}$ and variance $\sigma_{\text {disp }}^{2} . \lambda$ and $X_{j}$ are also independent random variables for all $j$. Let us determine the mean and the variance of $S$ but also the asymptotic form of the distribution of $S$ when $\lambda \rightarrow+\infty$. If we apply the law of total expectation, the mean of $S, \mathbb{E}(S)$ is given by

$$
\mathbb{E}(\mathrm{S})=\mathbb{E}\left(\mathbb{E}\left(\mathrm{S} \mid \lambda_{i}\right)\right)=\sum_{n=0}^{+\infty} \mathbb{E}\left(\mathrm{S} \mid \lambda_{i}=n\right) \mathbb{P}\left(\lambda_{i}=n\right)
$$

Where $\mathbb{E}\left(\mathrm{S} \mid \lambda_{i}\right)$ is the mean of $S$ conditionally to $\lambda_{i}$. As we have also

$$
\mathbb{E}\left(\mathrm{S} \mid \lambda_{i}=n\right)=\mathbb{E}\left(\sum_{j=1}^{n} \mathrm{X}_{\mathrm{j}}\right)=n \mathbb{E}(X)=n \mu_{\text {disp }}
$$

We can conclude that the expression of the mean of $S$ is

$$
\mathbb{E}(\mathrm{S})=\sum_{n=0}^{+\infty} n \mu_{\text {disp }} \mathbb{P}\left(\lambda_{i}=n\right)=\lambda \mu_{\text {disp }}
$$

since $\lambda$ is the mean of $\lambda_{i}$.

The law of total variance states that the variance of $S, \mathbb{V}(S)$ is equal to

$$
\mathbb{V}(S)=\mathbb{E}\left(\mathbb{V}\left(\mathrm{S} \mid \lambda_{i}\right)\right)+\mathbb{V}\left(\mathbb{E}\left(\mathrm{S} \mid \lambda_{i}\right)\right)
$$

Where $\mathbb{V}\left(S \mid \lambda_{i}\right)$ is the variance of $S$ conditionally to $\lambda_{i}$. The first term $\mathbb{E}\left(\mathbb{V}\left(S \mid \lambda_{i}\right)\right)$ of equation 9 is easily derived as $X_{j}$ are i.i.d. random variables,

$$
\mathbb{V}\left(\mathrm{S} \mid \lambda_{i}=n\right)=\mathbb{V}\left(\sum_{j=1}^{n} \mathrm{X}_{\mathrm{j}}\right)=\sum_{j=1}^{n} \mathbb{V}\left(X_{j}\right)=n \sigma_{\text {disp }}^{2}
$$

and thus

$$
\mathbb{E}\left(\mathbb{V}\left(\mathrm{S} \mid \lambda_{i}\right)\right)=\mathbb{E}\left(\lambda_{i} \sigma_{\text {disp }}^{2}\right)=\lambda \sigma_{\text {disp }}^{2}
$$

The second component of equation $9, \mathbb{V}\left(\mathbb{E}\left(S \mid \lambda_{i}\right)\right)$, is just

It follows that

$$
\mathbb{V}\left(\mathbb{E}\left(\mathrm{S} \mid \lambda_{i}\right)\right)=\mathbb{V}\left(\lambda_{i} \mu_{\text {disp }}\right)=\lambda \mu_{\text {disp }}^{2}
$$

$$
\mathbb{V}(\mathrm{S})=\lambda \sigma_{\text {disp }}^{2}+\lambda \mu_{\text {disp }}^{2}
$$

Let us consider the random variable $Z$,

$$
Z=\frac{\mathrm{S}-\lambda \mu_{\text {disp }}}{\sqrt{\lambda \sigma_{\text {disp }}^{2}+\lambda \mu_{\text {disp }}^{2}}}
$$

and determine its moment-generation function (MGF) $M_{Z}$,

$$
M_{Z}(t)=\mathbb{E}(\exp (t Z)), t \in \mathbb{R}
$$

The MGF of $S$ is defined by

$$
M_{\mathrm{S}}(t)=\mathbb{E}(\exp (t S))=\mathbb{E}\left(\mathbb{E}\left(\exp \left(t\left(S \mid \lambda_{i}\right)\right)\right)\right), t \in \mathbb{R}
$$

Conditionally to $\lambda_{i}=n$, we have 


$$
\begin{aligned}
\mathbb{E}\left(\exp \left(t\left(S \mid \lambda_{i}\right)\right)\right) & =\mathbb{E}\left(\exp \left(t \sum_{j=1}^{n} X_{j}\right)\right) \\
& =\mathbb{E}\left(\prod_{j=1}^{n} \exp \left(t X_{j}\right)\right)=M_{\mathrm{X}}(t)^{n}
\end{aligned}
$$

as $X_{j}$ are i.i.d. random variables and where $M_{X}$ is the MGF of the random variable $X$. The MGF of $\mathrm{S}$ is then

$$
\begin{aligned}
M_{\mathrm{S}}(t)=\mathbb{E}\left(M_{X}(t)^{\lambda_{i}}\right) & =\sum_{n=0}^{+\infty} M_{\mathrm{X}}(t)^{n} \mathbb{P}\left(\lambda_{i}=n\right) \\
& =\sum_{n=0}^{+\infty} \frac{\exp (-\lambda)\left(M_{\mathrm{X}}(t) \lambda\right)^{n}}{n !} \\
& =\exp \left(\lambda M_{X}(t)-\lambda\right), t \in \mathbb{R}
\end{aligned}
$$

It follows that

$$
\begin{array}{r}
M_{Z}(t)=\exp \left(\lambda M_{X}\left(\frac{t}{\sqrt{\lambda \sigma_{\text {disp }}^{2}+\lambda \mu_{\text {disp }}^{2}}}\right)-\lambda\right. \\
\left.-\frac{\lambda \mu_{\text {disp }} t}{\sqrt{\lambda \sigma_{\text {disp }}^{2}+\lambda \mu_{\text {disp }}^{2}}}\right), t \in \mathbb{R}
\end{array}
$$

The series expansion of $M_{\mathrm{X}}$ can be used here if the different moments of $X$ can be defined

$$
\begin{aligned}
M_{\mathrm{X}}(t)= & 1+t \mathbb{E}(X)+\frac{t^{2} \mathbb{E}\left(\mathrm{X}^{2}\right)}{2 !}+\cdots+\frac{t^{j} \mathbb{E}\left(\mathrm{X}^{\mathrm{j}}\right)}{j !}+\cdots \\
& M_{\mathrm{X}}(t)=1+t<D B G 4> \\
+ & \frac{t^{2}\left(\sigma_{\text {disp }}^{2}+\mu_{\text {disp }}^{2}\right)}{2 !}+\frac{t^{3} \mathbb{E}\left(\mathrm{X}^{3}\right)}{3 !}+\cdots
\end{aligned}
$$

and thus

$$
\begin{aligned}
& M_{\mathrm{X}}\left(\frac{t}{\sqrt{\lambda \sigma_{\text {disp }}^{2}+\lambda \mu_{\text {disp }}^{2}}}\right)=1+\frac{t \mu_{\text {disp }}}{\sqrt{\lambda \sigma_{\text {disp }}^{2}+\lambda \mu_{\text {disp }}^{2}}} \\
& +\frac{t^{2}}{2 \lambda}+\frac{\left(\frac{t}{\sqrt{\lambda \sigma_{\text {disp }}^{2}+\lambda \mu_{\text {disp }}^{2}}}\right)^{3} \mathbb{E}\left(\mathrm{X}^{3}\right)}{3 !}+\cdots
\end{aligned}
$$

When $\lambda \rightarrow+\infty$, we get

$$
\begin{gathered}
M_{\mathrm{X}}\left(\frac{t}{\sqrt{\lambda \sigma_{\text {disp }}^{2}+\lambda \mu_{\text {disp }}^{2}}}\right) \\
=1+\frac{t \mu_{\text {disp }}}{\sqrt{\lambda \sigma_{\text {disp }}^{2}+\lambda \mu_{\text {disp }}^{2}}}+\frac{t^{2}}{2 \lambda}+0\left(\frac{1}{\lambda}\right), t \in \mathbb{R}
\end{gathered}
$$

The MGF of the random variable $Z$ of Eq. (20) is then simplified when $\lambda \rightarrow+\infty$ in the following way

$$
M_{Z}(t)=\exp \left(\frac{t^{2}}{2}+0(1)\right), t \in \mathbb{R}
$$

The term $\exp \left(\frac{t^{2}}{2}\right)$ corresponds to the MGF of a standard normal distribution. Consequently, when $\lambda \rightarrow+\infty, Z$ converges to a standard normal distribution and $S$ converges to a normal distribution of mean $\lambda \mu_{\text {disp }}$, variance $\lambda \sigma_{\text {disp }}^{2}+$ $\lambda \mu_{\text {disp }}^{2}$

\section{B. Relation between method 1 and 2}

This demonstration proves that the method 1 and 2 have the same mean and the same variance. In the method 1 , the random variable $S$ does not follow a usual law. But in the method 2, the random variable follows a Gaussian law. Let us determine the mean and the variance of $S$, as used in the method 1, equal to $\lambda_{i} \times X$ where $\lambda_{i}$ follows a Poisson distribution with mean $\lambda$ and the random variable $X$ follows a normal distribution with mean $\mu_{\text {disp }}$ and variance $\frac{\sigma_{d i s p}^{2}}{\lambda_{i}}$. One has with the law of total expectation:

$$
\begin{gathered}
\mathbb{E}(S)=\mathbb{E}\left(\mathbb{E}\left(S \mid \lambda_{i}\right)\right)=\mathbb{E}\left(\mathbb{E}\left(\lambda_{i} \times X \mid \lambda_{i}\right)\right) \\
=\mathbb{E}\left(\lambda_{i} \times \mathbb{E}\left(X \mid \lambda_{i}\right)\right)=\mathbb{E}\left(\lambda_{i} \times \mu_{\text {disp }}\right)=\lambda \mu_{\text {disp }}
\end{gathered}
$$

where $\mathbb{E}\left(S \mid \lambda_{i}\right)$ is the mean of $S$ conditionally to $\lambda_{i}$. The law of total variance states that the variance of $S, \mathbb{V}(S)$, is equal to

$$
\mathbb{V}(S)=\mathbb{E}\left(\mathbb{V}\left(S \mid \lambda_{i}\right)\right)+\mathbb{V}\left(\mathbb{E}\left(S \mid \lambda_{i}\right)\right)
$$

where $\mathbb{V}\left(S \mid \lambda_{i}\right)$ is the variance of $S$ conditionally to $\lambda_{i}$. The first term $\mathbb{E}\left(\mathbb{V}\left(S \mid \lambda_{i}\right)\right)$ corresponds to

$$
\mathbb{V}\left(S \mid \lambda_{i}\right)=\mathbb{V}\left(\lambda_{i} \times X \mid \lambda_{i}\right)=\lambda_{i}^{2} \mathbb{V}\left(X \mid \lambda_{i}\right)=\lambda_{i} \sigma_{\text {disp }}^{2}
$$
and thus

$$
\mathbb{E}\left(\mathbb{V}\left(S \mid \lambda_{i}\right)\right)=\mathbb{E}\left(\lambda_{i} \sigma_{\text {disp }}^{2}\right)=\lambda \sigma_{\text {disp }}^{2}
$$

The second component of variance, $\mathbb{V}\left(\mathbb{E}\left(S \mid \lambda_{i}\right)\right)$, is just

It follows that

$$
\mathbb{V}\left(\mathbb{E}\left(S \mid \lambda_{i}\right)\right)=\mathbb{V}\left(\lambda_{i} \mu_{\text {disp }}\right)=\lambda \mu_{\text {disp }}^{2}
$$

$$
\mathbb{V}(S)=\lambda \sigma_{\text {disp }}^{2}+\lambda \mu_{\text {disp }}^{2}
$$

In the method 1 , the random variable $S$ is defined by $\lambda_{i} \times X$, with $\lambda_{i}$ drawn in the distribution $D 1$ and $X$ drawn in the distribution $D 2$. We have shown here that the mean and the variance of the distribution of this random variable are the same as those of the distribution $D 3$.

\section{REFERENCES}

[1] G.R. Hopkinson, C.J. Dale, and P.W. Marshall, "Proton Effects in Charge Coupled Devices, "IEEE Trans. Nucl. Sci., vol. 43, no. 2, pp. 614-627, April 1996.

[2] C.J. Dale, L. Chen, P.J. McNulty, P.W. Marshall, and E.A. Burke, "A Comparison of Monte Carlo and Analytic Treatments of Displacement Damage in Si Microvolumes, "IEEE Trans. Nucl. Sci., vol. 41, no. 6, pp. 1974-1983, December 1994.

[3] C. Inguimbert, T. Nuns, M. C. Ursule, D. Falguère, D. Hervé, M. Beaumel, M. Poizat, "Modeling the Dark Current Non Uniformity of image sensors with GEANT4, "IEEE Trans. Nucl. Sci., vol. 61, no. 6, pp. 3323-3330, December 2014.

[4] M.C. Ursule, C. Inguimbert, and T. Nuns, "Impact of the Border Crossing Effects on the DCNU for Pixel Arrays Irradiated With High Energy Protons, "

IEEE Trans. Nucl. Sci., vol. 63, no. 4, pp. 2159-2167, August 2016.

[5] C.J. Dale, P.W. Marshall, E.A. Burke, G.P. Summers, and G.E. Bender, "The Generation Lifetime Damage Factor and Its Variance in Silicon, " IEEE Trans. Nucl. Sci., vol. 36, no. 6, pp. 1872-1881, December 1989. 
[6] P.W. Marshall, C.J. Dale, E.A. Burke, G.P. Summers, and G.E. Bender, "Displacement Damage Extremes in Silicon Depletion Regions, " IEEE Trans. Nucl. Sci., vol. 36, no. 6, pp. 1831-1839, December 1989.

[7] P.W. Marshall, C.J. Dale, and E.A. Burke, "Proton-Induced Displacement Damage Distributions and Extremes in Silicon Microvolumes, " IEEE Trans. Nucl. Sci., vol. 37, no. 6, pp. 1776-1783, December 1990.

[8] M.S. Robbins, "High-Energy Proton-Induced Dark Signal in Silicon Charge Coupled Devices, "IEEE Trans. Nucl. Sci., vol. 47, no. 6, pp. 2473 2479, December 2000.

[9] J. Bogaerts, B. Dierickx, and R. Mertens, "Enhanced dark current generation in proton-irradiated CMOS active pixel sensors," IEEE Trans. Nucl. Sci., vol. 49, no. 3, pp. 1513-1521, June. 2002.

[10] R. Germanicus, S. Barde, L. Dusseau, G. Rolland, C. Barillot, F. Saigne, R. Ecoffet, P. Calvel, J. Fesquet, J. Gasiot, "Evaluation and prediction of the degradation of a COTS CCD induced by displacement damage, " IEEE Trans. Nucl. Sci., vol. 46, no. 6, pp. 2830-2835, December 2002.

[11] C. Virmontois, V. Goiffon, P. Magnan, S. Girard, C. Inguimbert, S.Petit, G. Rolland, and O. Saint-Pe, "Displacement damage effects due to neutron and proton irradiations on CMOS image sensors manufactured in deep submicron technology," IEEE Trans. Nucl. Sci., vol. 57, no. 6, pp. 3101-3108, December 2010

[12] J. M. Belloir, V. Goiffon, C. Virmontois, M. raine, P. Paillet, O. Duhamel, M. galliardin, R. Molina, P. Magnan, O. Gilard, "Pixel pitch and particle energy influence on the dark current distribution of neutron irradiated CMOS image sensors, " Optic Express, vol. 24, no. 4, pp. 4299-4315, February 2016

[13] George J. Schlenker, "Methods for calculating the probability distribution of sums of independent random variables, " ADA170465, Final rept., Army armament munitions and chemical command rock island il systems analysis office, July 1986.

[14] e2v, [Online].Available:http://www.e2v-us.com, Accessed Mar. 14, 2017.

[15] Insoo Jun, M. Xapsos, S. R. Messenger, E. A. Burke, R. Walters "Proton non ionising energy loss (NIEL) for device applications, "IEEE Trans. Nucl. Sci., vol. 50, no. 6, pp. 1924-1931, December. 2003.

[16] S. Agostinelli and et al., "GEANT4 a simulation toolkit," Nucl. Instrum. Methods Phys. Rev. A, vol. A506, pp. 250-303, 2003.

[17] J. Allison and et al., "GEANT4 Developments and Applications," IEEE Trans. Nucl. Sci., vol. 53, no. 1, pp. 270-278, February 2006.

[18] J. R. Srour, "Universal Damage Factor for Radiation-Induced Dark Current in Silicon Devices," IEEE Trans. Nucl. Sci., vol. 47, no. 6, pp. $2451-$ 2459, December 2000.

[19] C. Inguimbert, R. Gigante, "NEMO: A Code to Compute NIEL of Protons, Neutrons, Electrons, and Heavy Ions, " IEEE Trans. Nucl. Sci., vol. 53, no. 4, pp. 1967-1972, August 2006.

[20] E. Martin, O. Gilard, T. Nuns, J.P. David and C. Virmontois, "Prediction of CMOS Image Sensor Dark Current Distribution and Noise in a Space Radiation Environment, "IEEE Trans. Nucl. Sci, vol. 60, no. 3, pp. 22802287, June 2013

[21] Ziegler J. F., Biersack J. P. and Littmark U.," The stopping and range of Ions in solids. volume 1," Pergamon Press, New-York, 1985.

[22] V. E. Bening and V. Y. Korolev, "Generalized Poisson Models and their Applications in Insurance and Finance, " VSP International Science Publishers, Ed., 2002.

[23] S. Kullback et R. Leibler, "On information and sufficiency, " Annals of Mathematical Statistics, vol. 22, p. 79-86, 1951. 